\title{
IMAGING INSIGHTS IN EISENMENGER'S SYNDROME: FROM BASICS TO SOPHISTICATION
}

Srilakshmi Adhyapak ${ }^{1}$, Kiron Varghese ${ }^{2}$, Preethu Anand ${ }^{3}$, Tarun Rao ${ }^{3}$, and Jabraan Shaikh $^{3}$

${ }^{1}$ St. Johm's Medical College Hospital

${ }^{2}$ St. John's Medical College Hospital

${ }^{3}$ St John's Medical College Hospital

July 16, 2020

\begin{abstract}
We describe an unusual case of a large Aorto-pulmonary window which presented as Eisenmenger's syndrome at the age of 20 years in a male patient. We take this opportunity to highlight some of the basic investigations like Chest X Ray and Contrast Echocardiography which can delineate the level of the shunt. Attention should be given to the subtle bedside clinical signs which can give clues to the diagnosis. We did a cardiac CT scan for confirmation of the diagnosis.
\end{abstract}

IMAGING INSIGHTS IN EISENMENGER'S SYNDROME: FROM BASICS TO SOPHISTICATION

Department of Cardiology; St. John's medical College Hospital, India

Srilakshmi M Adhyapak DNB

Associate Professor, Dept of Cardiology

St. John's Medical College Hospital, India.

Dr Preethu Anand MBBS

Junior Resident, Department of Cardiology

Dr Tarun Rao MD

Senior Resident, Department of Cardiology

Dr Jabraan U Shaikh MD

Senior Resident, Department of Cardiology

Kiron Varghese MD, DM

Head and Professor of Cardiology

St. John's Medical College Hospital, India.

No Funding. No conflict of Interest .

Word Count : Total: 1189 
Address for Correspondence : Srilakshmi M Adhyapak, Associate Prof, Dept of Cardiology, St. John's Medical College Hospital, Bangalore 560034, India, Tel: 91-80-22065615, Fax: 91-80-25630703. Email: srili2881967@yahoo.com

\begin{abstract}
:
We describe an unusual case of a large Aorto-pulmonary window which presented as Eisenmenger's syndrome at the age of 20 years in a male patient. We take this opportunity to highlight some of the basic investigations like Chest X Ray and Contrast Echocardiography which can delineate the level of the shunt. Attention should be given to the subtle bedside clinical signs which can give clues to the diagnosis. We did a cardiac CT scan for confirmation of the diagnosis.
\end{abstract}

Eisemenger's syndrome is a syndrome complex constituting reversal of large left to right shunts with increasing pulmonary vascular resistance. These shunts can be at the atrial septum, ventricular septum or at the great arterial levels.

Certain subtle clinical features have been defined as hallmarks of individual lesions, mainly involving the second heart sound. A single second sound is a feature of reversal of flow through a ventricular septal defect, while a normally split second sound features reversal of flow through a patent ductus arteriosus (PDA). In an atrial septal defect(ASD), reversal of flow may shorten the gap between the two components of the second heart sound with a fixed split. The split in the second heart sound remains fixed although it may shorten[1].

Differential cyanosis is a feature of reversal of flow in a PDA. ASD and VSD usually exhibit uniform pandigital cyanosis and clubbing. The cardiac size usually regresses with reversal of flow in a ventricular septal defect (VSD), while in an ASD, cardiomegaly persists.

The chest X Ray may feature subtle signs, which may help diagnosis further. Peripheral pruning of pulmonary vasculature with enlarged prominent main pulmonary artery is a sign of Eisenmenger's syndrome. The presence of cardiomegaly may help distinguish a pre-tricuspid shunt (ASD) while normal cardiac diameter signifies a post-tricuspid shunt which can be either a VSD or PDA.

A contrast Echocardiogram can define the anatomical position of the shunt. Injection of agitated saline into the ante-cubital vein can define the right to left shunt when the bubbles appear in the left heart after 5 to 7 cardiac cycles. In the aorta, if the bubbles appear in the descending aorta, the reversal of flow is through a PDA, while if the bubbles appear in the ascending aorta, the reversed flow is through an aorto-pulmonary window.

We wish to highlight these basic teaching pearls in a patient with Eisenmenger's syndrome and further describe this unusual case of an Aorto-pulmonary window presenting with Eisenmenger's syndrome at the age of 20 years .

We describe a 20 year old male who presented to us with 2 to 3 episodes of hemoptysis per day of $10 \mathrm{ml}$ each since 1 week. He had a childhood history of breathlessness and failure to thrive which was noticed when he was 5 months of age. He was hospitalized for the same and administered medications. The details of which were not available. Further, he had history of breathlessness on exertion at the age of 5 years due to which he could not play and keep up with his peers in out door activities. Besides, he developed repeated lower respiratory tract infections requiring hospitalization and systemic antibiotics. After the age of 14 years, the episodes of productive cough and breathlessness decreased. The clinical history of heart failure in childhood which regressed during adolescence in this 20 year old patient was a marker of this syndrome. Clinical examination revealed a right ventricular apex, left parasternal heave, loud P2 and a long early diastolic murmur along his left parasternal region. He had uniform pan-digital cyanosis and clubbing.

His Chest X Ray ( PA view) demonstrated that the cardiac apex was formed by the right ventricle, with an aneurismal main pulmonary artery segment. There was peripheral pruning of pulmonary vasculature. An interesting observation was that the right pulmonary artery was conspicuous by its absence, while a 
branching pulmonary artery was seen through the cardiac silhouette. The aortic arch was right sided as it coursed to the right of the vertebral column (Figure 1).

The 2D Echocardiography demonstrated a dilated right atrium, right ventricle and main pulmonary artery, with normal valves and normal biventricular function. There was no ASD, VSD or PDA (Figures 2,3 ).

A contrast echocardiography with agitated saline injected into the ante-cubital vein, showed contrast bubbles in the ascending aorta after 5 cardiac cycles. The contrast bubbles were not seen in the descending aorta, left ventricle or left atrium (Figure 4).

The clinical features and basic investigations like the chest X-ray and contrast Echocardiography demonstrated reversal of flow across an aorto-pulmonary window.

Further, a cardiac CT scan was done which confirmed a Type1 aorto-pulmonary window (Figures 5 to 11).

We wish to emphasize the importance of bedside clinical examination and basic imaging techniques which are still relevant in their contribution to the diagnosis of various clinical conditions.

\section{REFERENCES :}

Paulwood. The Eisenmenger syndrome. Br Med J. 1958 Sep 20; 2(5098): 701-709.

\section{FIGURE LEGENDS:}

Figure 1: A Chest X Ray ( PA view) demonstrates cardiomegaly. The cardiac silhouette forms an apex formed by the right ventricle, with the aneurismal main pulmonary artery segment. There is peripheral pruning of pulmonary vasculature. An interesting observation is that the right pulmonary artery is conspicuous by its absence, while a branching pulmonary artery is seen through the cardiac silhouette. The aortic arch is right sided as it courses to the right of the vertebral column.

Figure 2 : This is an apical 4 chamber view on 2D Echocardiography demonstrating a dilated right atrium and right ventricle. The color flow is laminar across both atrio-ventricular valves with no evidence of atrio ventricular valve stenosis or regurgitation. The inter-atrial septum and inter-ventricular septum are intact.

Figure 3: This is the parasternal short axis view ( SAX) on 2D Echocardiography demonstrating the aorta and enlarged pulmonary artery with normal relation of the great arteries.

Figure 4 : This is a suprasternal view of the aortic arch. This is a contrast echocardiography with agitated saline injected through the ante-cubital vein. The contrast bubbles are seen in the ascending aorta after 5 cardiac cycles.

Figure 5: This is a CT scan at the base of the heart demonstrating a large Type 1 Aorto-Pulmonary window. The short main pulmonary artery with branch pulmonary arteries arising from the aorta is visualized.

Figure 6 : The CT scan demonstrates the right sided thoracic aortic arch.

Figure $\mathbf{7 , 8 , 9 , 1 0 , 1 1}$ : These reconstructed CT scan images demonstrate the large Type 1 Aorto-Pulmonary window arising from the ascending aorta, which then turns to continue as a right aortic arch. 


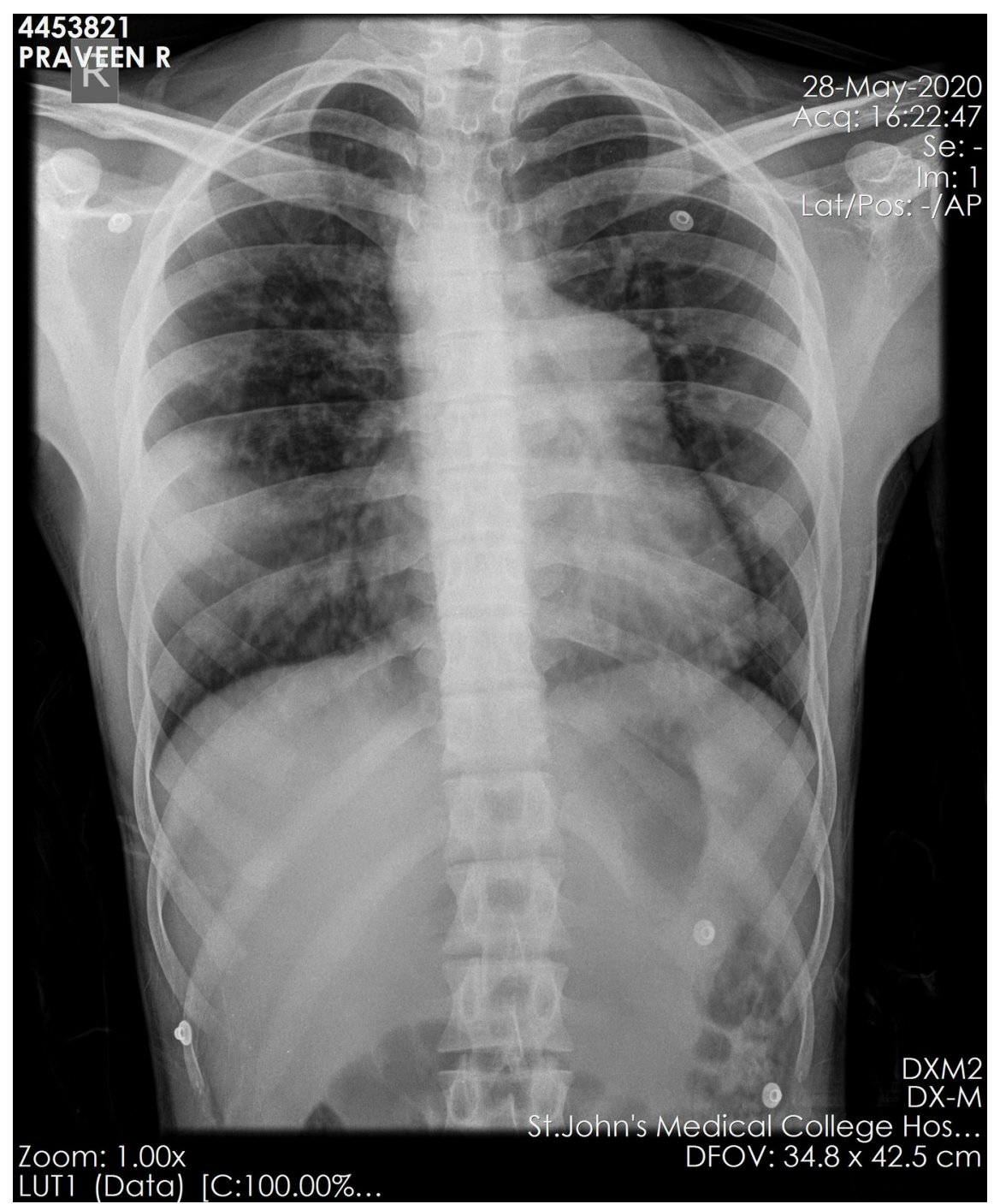




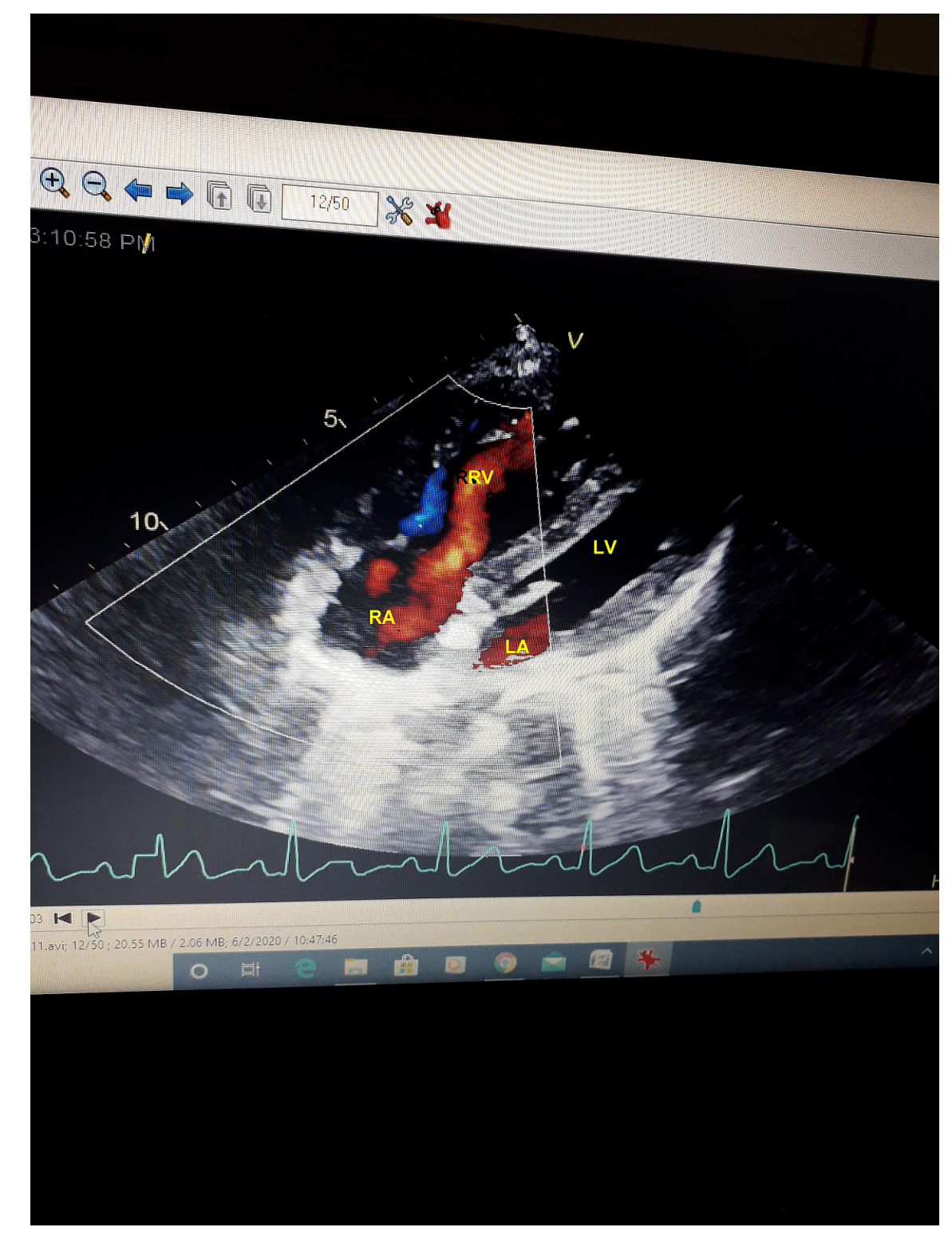




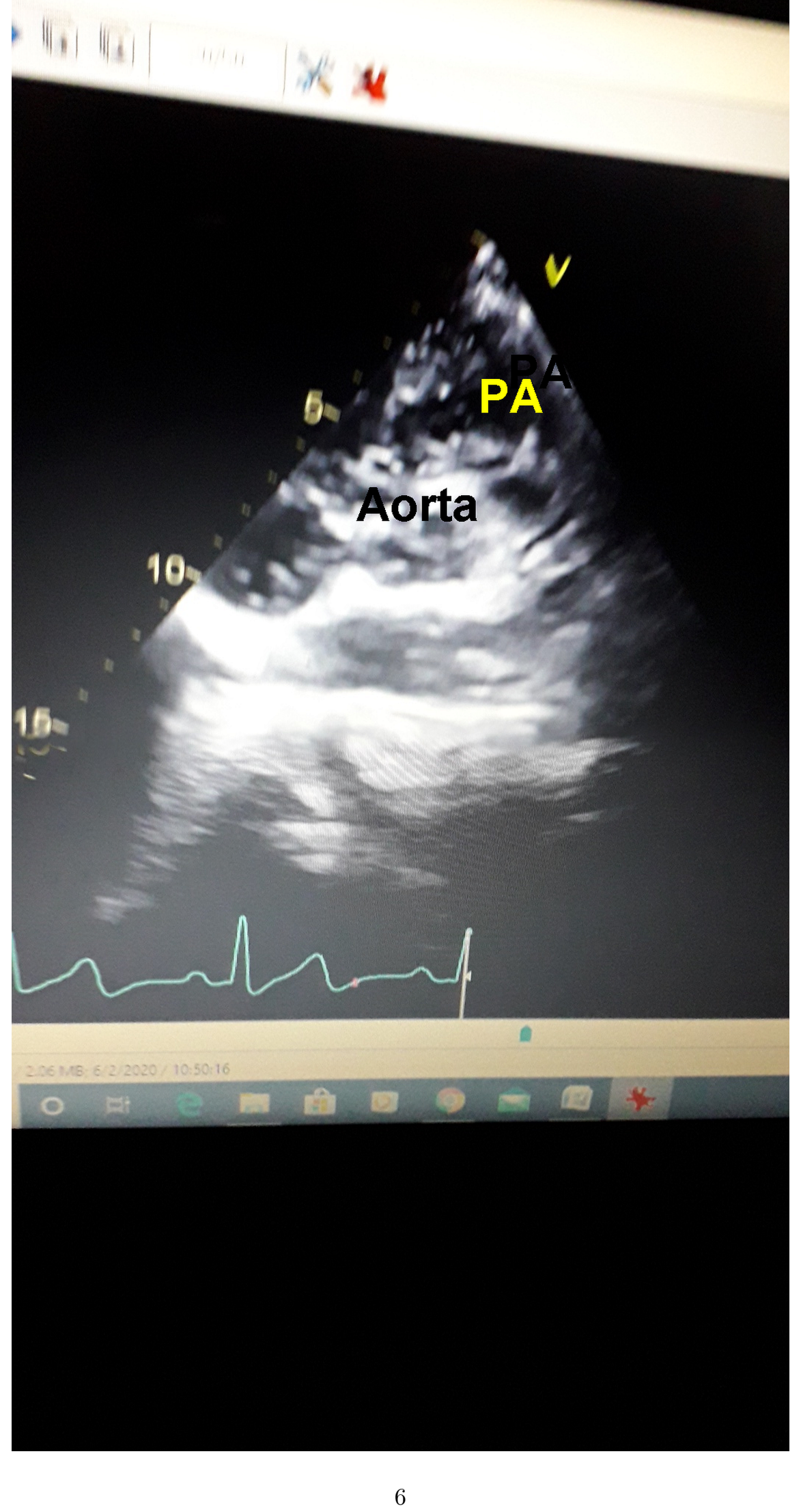




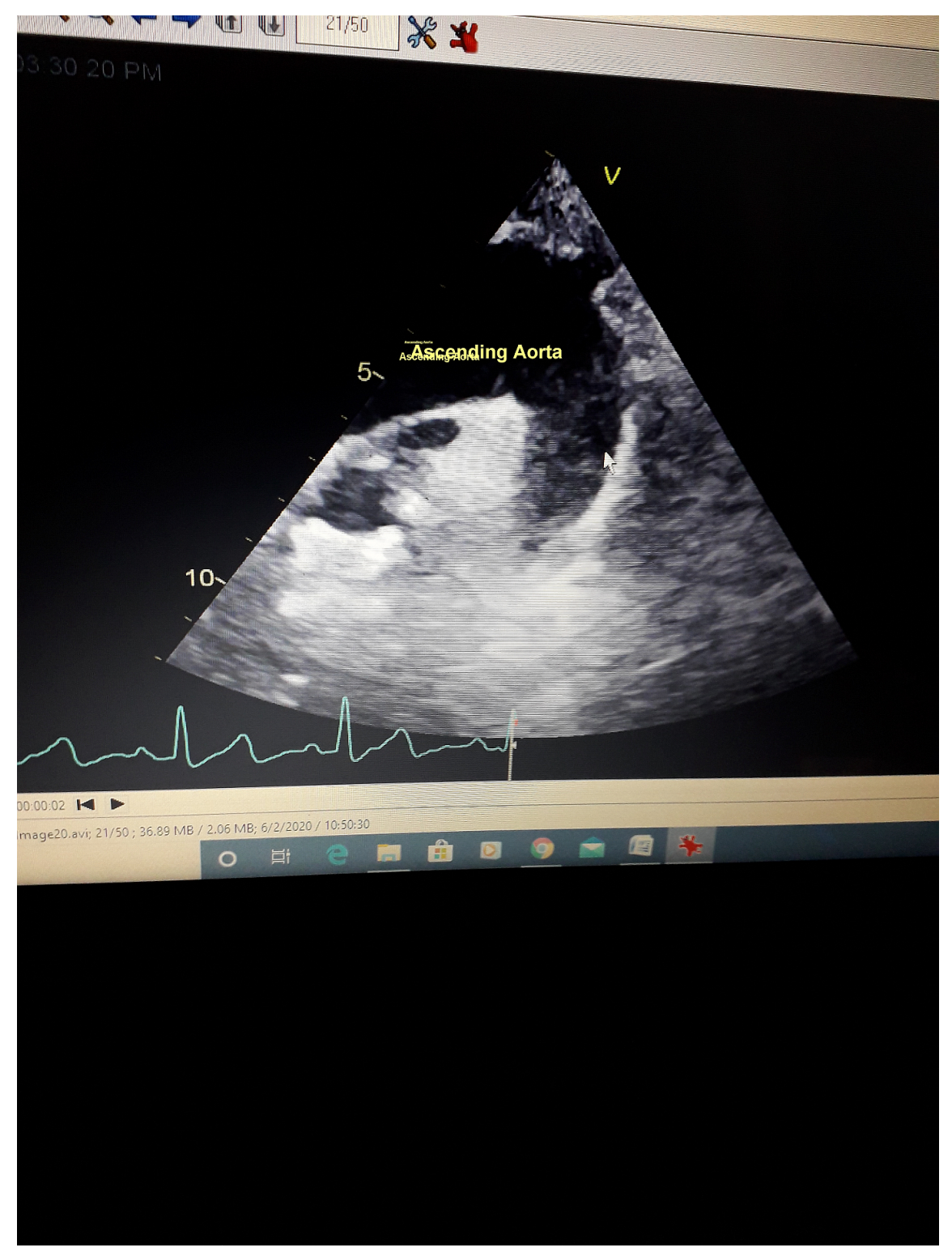



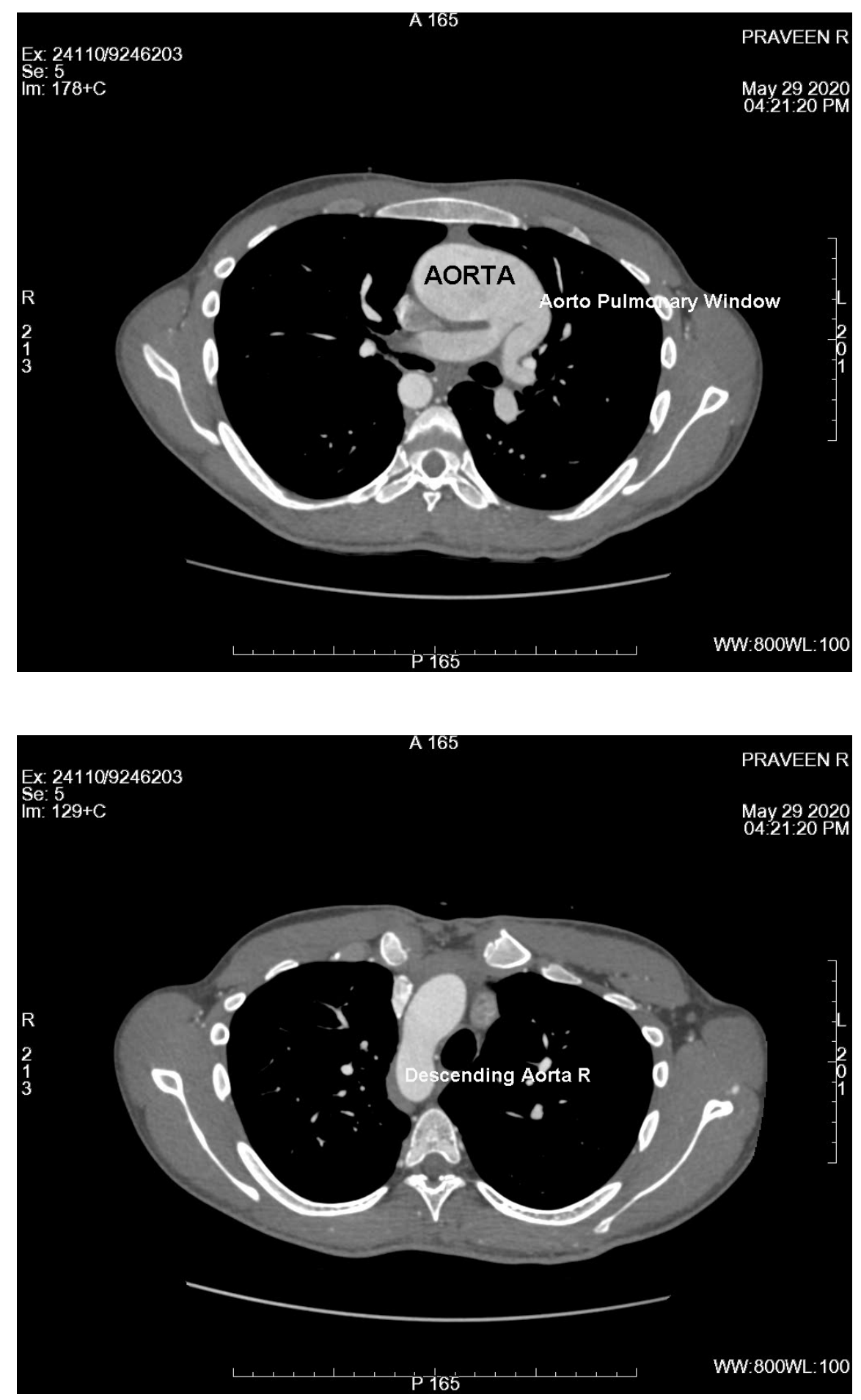

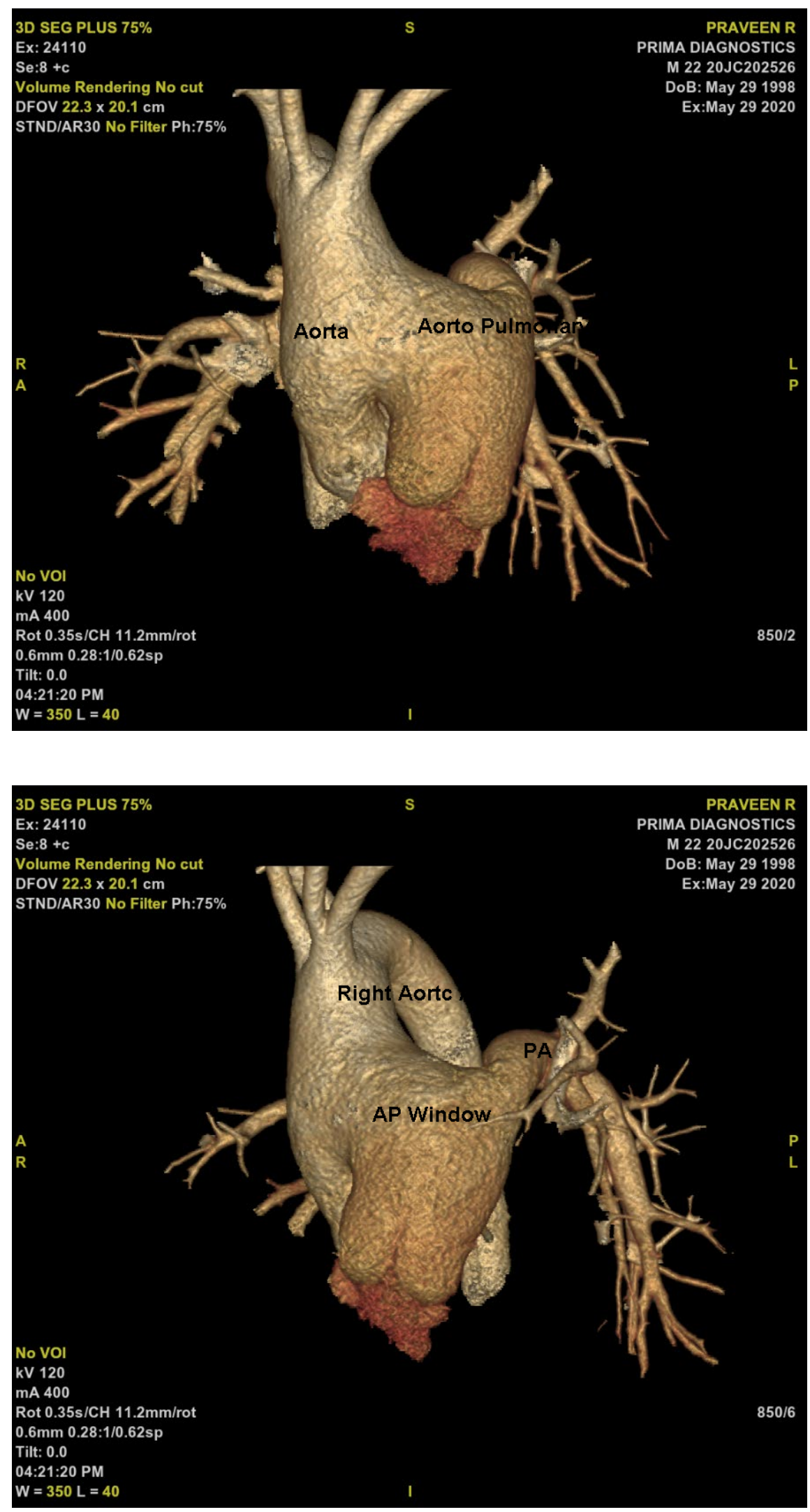

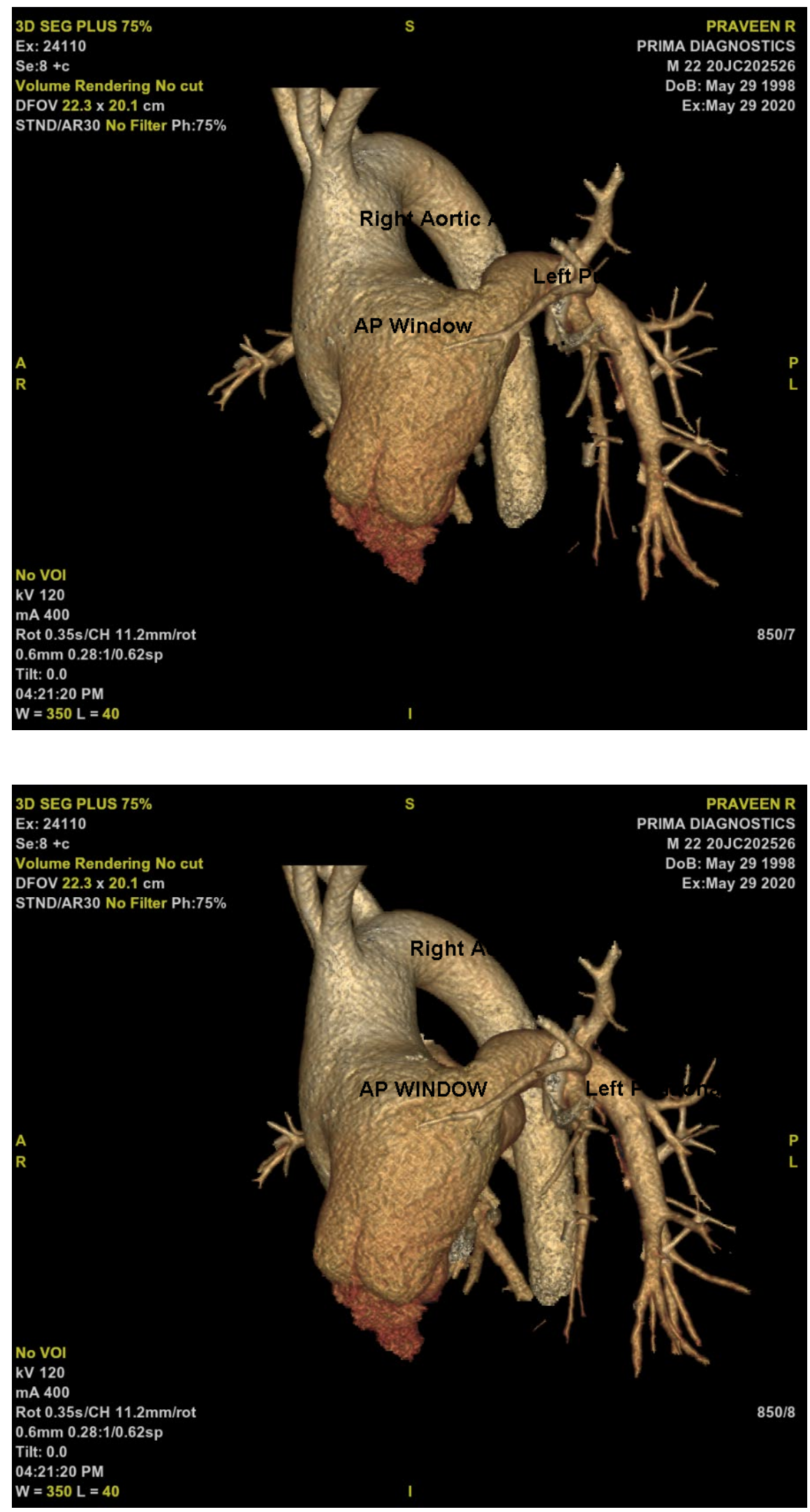


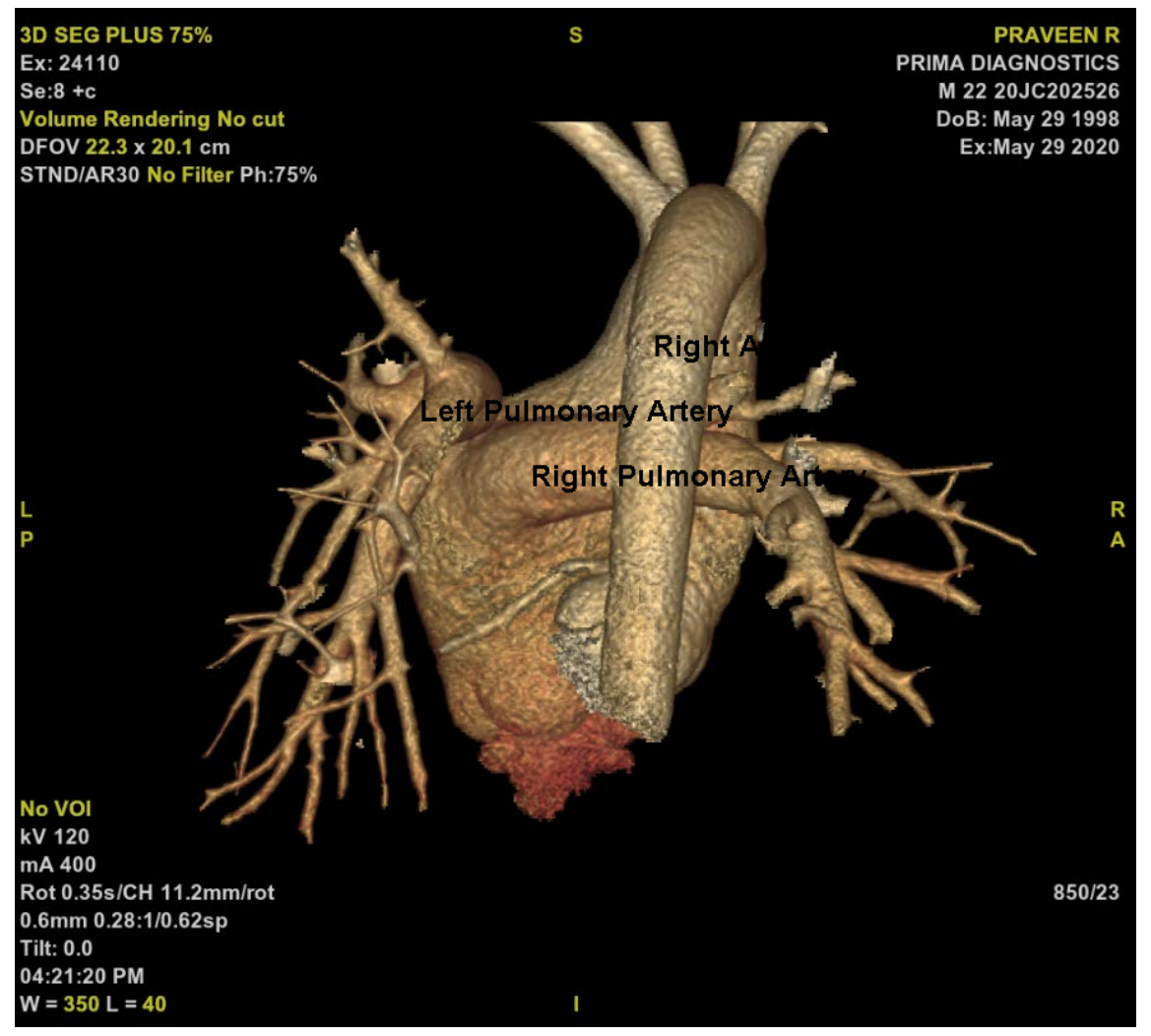

